\title{
Ultra-fast and compact all-optical encoder based on a photonic crystal nano-resonator without using nonlinear materials
}

\author{
Ahmad Mohebzadeh-Bahabady and Saeed Olyaee* \\ Nano-photonics and Optoelectronics Research Laboratory (NORLab), Faculty of Electrical Engineering, Shahid \\ Rajaee Teacher Training University (SRTTU), Lavizan, 16788-15811, Tehran, Iran
}

Received March 14, 2019; accepted March 30, 2019; published March 31, 2019

\begin{abstract}
In this paper an ultra-compact all-optical encoder is presented by using a two-dimensional photonic crystal. The designed logic gate is based on the interference effect. The proposed structure consists of several photonic crystal waveguides connected by 2 nanoresonators. The nano-resonators are designed to reduce the size of the radius of the dielectric rods. The contrast ratios and delay time for the proposed all-optical encoder are respectively $6 \mathrm{~dB}$ and $125 \mathrm{fs}$. The size of the structure is equal to $132 \mu \mathrm{m}^{2}$. Equality of the output power in the logic states "one", small dimensions, low delay time, compact and simple structure have shown that the logic gate is suitable for optical integrated circuits.
\end{abstract}

One of the goals of research in new communication networks is to achieve ultra-fast transmission speeds. For this purpose, all communication steps, such as sending, receiving, and processing information on the network, should be performed in a full optical form [1-2]. To realize these all-optical communication networks, alloptical devices are required for each sector. For this reason, all-optical devices have attracted the attention of many researchers. So far, all-optical devices have been introduced in a variety of platforms [3-5]. One of the platforms that is suitable for designing ultra-compact and extremely low-power optical circuits is the photonic crystal substrate [5-6]. The principle of the photonic band gap allows the designer to confine and control the light in ultra-small spaces. According to this principle, the light with a specific frequency which is in the band gap, does not propagate in the photonic crystal structure. To date, various optical devices such as all-optical filters [7], alloptical multiplexers [8], all-optical sensors [9-10], alloptical logic gates [11], and so on have been designed and presented.

An all-optical encoder is an all-optical logic gate with $2 n$ input and $n$ output ports. At any time, only one of the input ports is active and the other input ports are disabled [12]. Given the activation of the input, the binary code is generated at the output ports. This logic gate is used in channel encoding blocks of communication links and producing binary codes in optical analog-to-digital converters.

*E-mail: s_olyaee@sru.ac.ir
In 2015 , by placing two elliptical rings in a square photonic crystal structure, a 4 to 2 encoder was proposed [13]. Its mechanism is based on the use of nonlinear materials. The output power of over $40 \%$ of input power is considered to be the logical level of one, which is the low output intensity. Moniem presented a 4 to 2 encoder using 4 photonic crystal ring resonators [14]. In this structure, in addition to 4 input ports and 2 output ports, 2 input bias ports are considered. The ratio of the output power received to the input power is acceptable, but 2 sources of the bias input significantly increase the power consumption. The other 4 to 2 encoder is designed in a GaAs photonic crystal substrate [15]. In this structure, the minimum power required to start the encoder is $1 \mathrm{kw} / \mu \mathrm{m} 2$, which is high power compared to other structures. SeafDargahi has proposed an encoder using 4 ring resonators without using nonlinear materials [16]. The output power is different for logical level of one equivalent of 42 to 90 percent of input, which is considered to be the main fault of this structure.

In general, the mechanism of logic gates based on photonic crystals is divided into three categories [17-19]. The first method is the use of nonlinear materials in the photonic crystals structure. In this kind of logic gate, high contrast ratio is obtained, but the delay time is high and requires high input optical power. The second method is the interference effect. The destructive and constructive interference determines the amount of light transmitted to the output. In this category of logic gates, high input optical power is not needed, and delay time is less than the first method. In the third method, the self-collimation method is used.

The efficiency of optical logic gates is examined by several parameters. One of the parameters is contrast ratio. The contrast ratio represents the distinction between the logical level " 1 " and "0" in the received optical power [20]. This parameter is defined as:

$$
C R=10 \log \left(\frac{P_{\text {on }}}{P_{\text {off }}}\right)
$$

where $P_{\text {on }}$ is the optical power value for the logic " 1 " and $\mathrm{P}_{\text {off }}$ is the optical power for the logic "0". The contrast ratio is calculated in the worst performance conditions of 
the designed logic gate, so the lowest value of $\mathrm{P}_{\mathrm{on}}$ and the highest $\mathrm{P}_{\text {off }}$ is considered.

Another parameter for checking the efficiency of logic gate is the logic gate response time to the input signal (or delay time), which is a factor in the speed of data transmission by that logic gate [21]. The lower delay time will mean the higher data transmission speeds. Depending on the type of resonance used for design, the delay time will be different.

The fundamental photonic crystal is a two-dimensional photonic crystal hexagonal structure of repetition of cylindrical dielectric rods in an air substrate. The radius of the dielectric rods is equal to $115 \mathrm{~nm}$. The refractive index of the rods is 3.4757 , which is a refractive index of silicon at $1550 \mathrm{~nm}$. The choice of silicon as a material allows this piece to be used with other silicon-based components in optical integrated circuits. The distance between the centers of two neighboring rods, which is called the lattice constant, is equal to $640 \mathrm{~nm}$. The overall thickness of the rods is $220 \mathrm{~nm}$.

The band gap structure is calculated using the plane wave expansion (PWE) method. The band gap diagram is shown in Fig. 1.

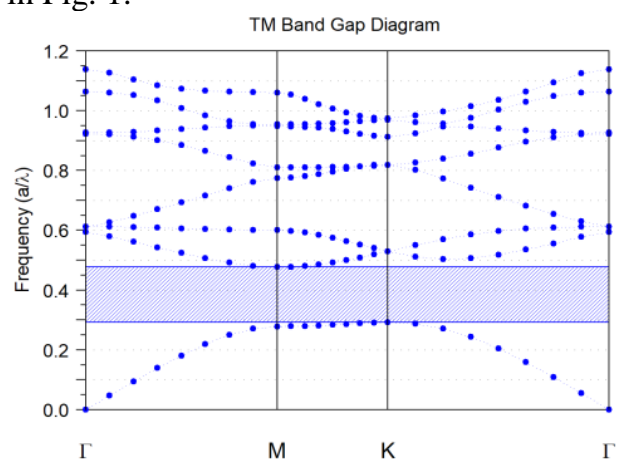

Fig. 1. TM band gap diagram of the photonic crystal used in the alloptical encoder (the photonic band gap lies between $1341 \mathrm{~nm}$ and $2191 \mathrm{~nm})$.

As shown in Fig. 1, the band gap of this structure ranges from $\mathrm{a} / \lambda=0.292$ to $\mathrm{a} / \lambda=0.477$. This range is limited to a wavelength of $1341 \mathrm{~nm}$ to $2191 \mathrm{~nm}$. From the transverse electric (TE) polarization and transverse magnetic (TM) polarization, the suitable polarization for the electromagnetic wave is the TM polarization.

The structure of the proposed all-optical encoder is shown in Fig. 2. The structure consists of several photonic crystal waveguides connected by two Nano-resonators. Four ports (I1 to I4) are designed for light input to the structure and two ports $(\mathrm{O} 1$ and $\mathrm{O} 2)$ to receive light from the structure. When input port I1 is active, both outputs must be zero. For this purpose, it is arranged that the waveguide connected to the port I1 is not connected to the output ports. As a result, this port can be considered at any point in the structure and can be changed depending on the use of the encoder. On the other hand, an output can be considered if needed. With this output, this state can be explained by the fact that the pair of inputs are zero. In the design, because the small size is important, this port is located between two output ports and connected to a short waveguide.

Nano-resonators are designed to reduce the size of the radius of the rods. One of the purposes for designing this all-optical encoder was that the output power was equal in different states. With the choice of a nano-resonator radius sized of $64 \mathrm{~nm}$, this goal was achieved.

To simulate the all-optical encoder function, each of the I1 to I4 ports is connected to a Gaussian optical source with a central wavelength of $1550 \mathrm{~nm}$.

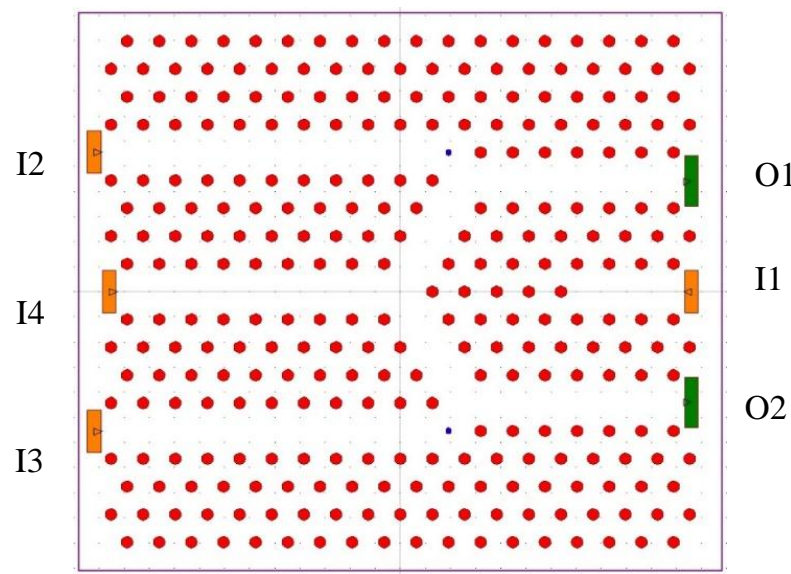

Fig. 2. The proposed photonic crystal structure to realize the all-optical encoder.

By placing two monitors in the $\mathrm{O} 1$ and $\mathrm{O} 2$ ports, the output power is calculated. According to the truth table of encoder, 4 states are considered. In each state, one of the inputs is active (at a logical value of one) and the other inputs are lacking optical power.

State 1: The optical power density at the port I1 is equal to $\mathrm{P}_{\mathrm{in}}=1 \mathrm{~mW} / \mu \mathrm{m}^{2}$, and in the other input ports, the optical power is zero. In this case, the input light is limited at the end of the waveguide and does not find a way to the output waveguides. As a result, there will be no optical power in both outputs and the power level is zero. The pattern of the electromagnetic field in the structure is shown in Fig. 3.

State 2: In this state, the $\mathrm{I} 2$ port's power is $\mathrm{P}_{\text {in }}$ and other inputs are zero. Figure 4 shows the electromagnetic field pattern of light in this case. As shown in Fig. 4, the input light passes through the nano-resonator 1 to the $\mathrm{O} 1$ output. Most of the light input goes to $\mathrm{O} 1$ and a small percentage of light is received at the $\mathrm{O} 2$. The output power of $\mathrm{O} 1$ is $0.461 \cdot \mathrm{P}_{\text {in }}$ and for $\mathrm{O} 2$ is $0.051 \cdot \mathrm{P}_{\text {in }}$.

State 3: In this state, only the power of the I3 is $\mathrm{P}_{\text {in }}$. In this state, the light enters the bottom input waveguide and transmits it through the nano-resonator 2 to the $\mathrm{O} 2$. The optical power received in this case is similar to state 2 . 
The optical power at $\mathrm{O} 1$ is $0.051 \cdot \mathrm{P}_{\text {in }}$ and at $\mathrm{O} 2$ is about $0.461 \cdot \mathrm{P}_{\text {in. }}$ Figure 5 shows the pattern of the electromagnetic field.

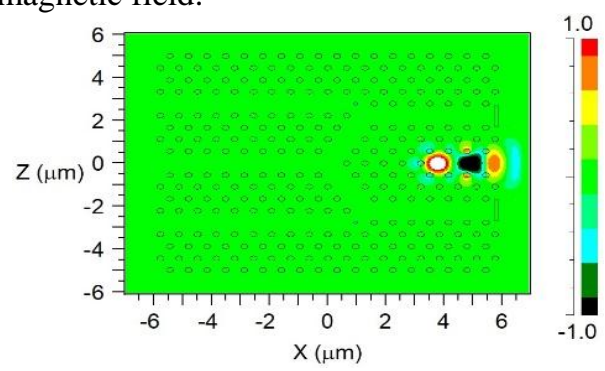

Fig. 3. The electromagnetic field pattern for the all-optical encoder when the input signal of I1 is one

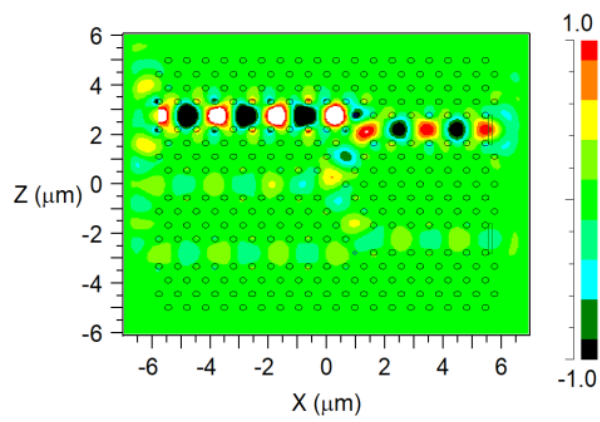

Fig. 4. The electromagnetic field pattern for the all-optical encoder when the input signal of $\mathrm{I} 2$ is one.

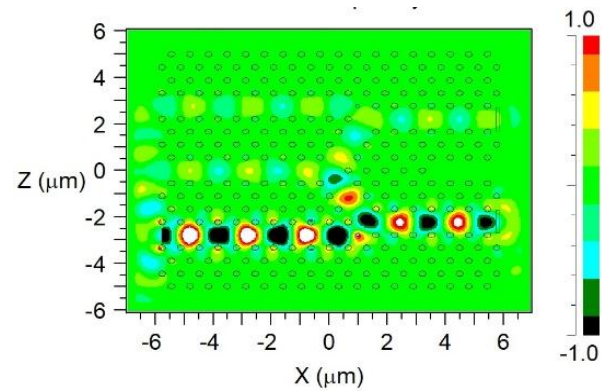

Fig. 5. The electromagnetic field pattern for the all-optical encoder when the input signal of $\mathrm{I} 3$ is one.

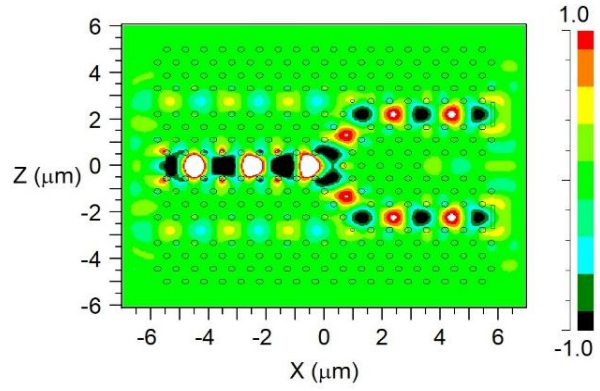

Fig. 6. The electromagnetic field pattern for the all-optical encoder when the input signal of I4 is one.

State 4: The input signal of I4 is active and the light enters the structure through the intermediate waveguide. As the electromagnetic field behavior is known, light passes through both nano-resonators to both output waveguides (Figure 6). In this case, the optical power in each output is $0.456 \cdot \mathrm{P}_{\text {in }}$.

These four modes are summarized in Table 1. Since no nonlinear material is used in the design of the structure, low input power is required. The size of the all-optical encoder is small and equal to $132 \mu \mathrm{m}^{2}$. The logic gate is only formed by placing two nano-resonators, which is a simple structure. These features make it possible to use this encoder in optical integrated circuits.

The delay time of this logic gate is obtained at $\mathrm{cT}=70$ $\mu \mathrm{m}$. Therefore, the delay time is obtained as $125 \mathrm{fs}$.

Table 1. Truth table and optical power in output for all-optical encoder.

\begin{tabular}{c|c|c|c|c|c}
\hline I1 & I2 & I3 & I4 & O1 & O2 \\
\hline \hline $\mathrm{P}_{\text {in }}$ & 0 & 0 & 0 & 0 & 0 \\
\hline 0 & $\mathrm{P}_{\text {in }}$ & 0 & 0 & $0.461 \mathrm{P}_{\text {in }}$ & $0.051 \mathrm{P}_{\text {in }}$ \\
\hline 0 & 0 & $\mathrm{P}_{\text {in }}$ & 0 & $0.051 \mathrm{P}_{\text {in }}$ & $0.461 \mathrm{P}_{\text {in }}$ \\
\hline 0 & 0 & 0 & $\mathrm{P}_{\text {in }}$ & $0.456 \mathrm{P}_{\text {in }}$ & $0.456 \mathrm{P}_{\text {in }}$ \\
\hline
\end{tabular}

This work was supported by Shahid Rajaee Teacher Training University under the contract number 9085 .

\section{References}

[1] A. Salmanpour, Sh. Mohammadnejad, A. Bahrami, Opt. Quant. Electr. 47, 2249 (2015).

[2] S.C. Xavier, B.E. Carolin, A.P. Kabilan, W. Johnson, IET Optoelectr. 10, 142 (2016).

[3] Y. Miyoshi, K. Ikeda, H. Tobioka, T. Inoue, S. Namiki, K. Kitayama, Opt. Expr. 16, 2570 (2008).

[4] D.K. Gayen, A. Bhattachryya, T. Chattopadhyay, J.N. Roy, J. Lightwave Techn. 30, 3387 (2012).

[5] A. Mohebzadeh-Bahabady, S. Olyaee, IET Optoelectr. 12, 191 (2018).

[6] Z. Mohebbi, N. Nozhat, F. Emami, Opt. Comm. 355, 130 (2015).

[7] M. Mansouri-Birjandi, M. Ghadrdan, Phot. Nanostr. -Fundamen. Appl. 21, 44 (2016).

[8] H. Alipour-Banaei, S. Serajmohammadi, F. Mehdizadeh, J. Optoelectr. Advanced Mater. 17, 259 (2015).

[9] A. Mohebzadeh-Bahabady, S. Olyaee, H. Arman, Current Nanoscen. 13, 421 (2017).

[10] S. Olyaee, A. Mohebzadeh-Bahabady, Opt. Quant. Electron. 47, 1881 (2015)

[11] F. Parandin, R. Malmir, M. Naseri, A. Zahedi, Superlatt. Microstr. 113, 737 (2018)

[12] F. Mehdizadeh, M. Soroosh, H. Alipour-Banaei, IET Optoelectr. 11, 29 (2017).

[13] M. Hassangholizadeh-Kashtiban, R. Sabbaghi-Nadooshan, H. Alipour-Banaei, Optik 126, 2368 (2015).

[14] T.A. Moniem, J. Modern Optics 63, 735 (2016).

[15] S. Gholamnejad, M. Zavvari, Opt. Quant. Electr. 49, 302 (2017).

[16] H. Seif-Dargahi, Photon. Network Comm. 36(2), 272 (2018).

[17] S. Olyaee, M. Seifouri, A. Mohebzadeh-Bahabady, M. Sardari, Opt. Quant. Electr. 50, 12 (2018).

[18] C. J. Wu, C. P. Liu, Z. Ouyang, Applied Optics.51, 680 (2012).

[19] Y.C. Jiang, S.B. Liu, H.F. Zhang, X.K. Kong, Optics Comm. 348, 90 (2015).

[20] A. Salmanpour, S. Mohammadnejad, P.T. Omran, Opt. Quant. Electr. 47, 3689 (2015).

[21] E.H. Shaik, N. Rangaswamy, J. Comput. Electr. 17, 337 (2018). 